\title{
Improvements to Energy Resolution of an X-ray Energy Dispersive Spectrum by Deconvolution Using the Zero Strobe Peak
}

\author{
M. Watanabe and D.B. Williams \\ Dept. of Materials Science and Engineering, Lehigh University, Bethlehem, PA18015, USA
}

X-ray energy dispersive spectrometry (XEDS) is one of the most efficient and robust approaches to analyze materials. However, the poor energy resolution of an XEDS detector (typically $\sim 140$ $\mathrm{eV}$ at the $\mathrm{MnK} \alpha$ peak for a conventional $\mathrm{Si}(\mathrm{Li})$ detector) may cause serious overlaps of generated X-ray peaks and degrade the peak-to-background $(\mathrm{P} / \mathrm{B})$ ratio (and hence the analytical sensitivity). Since peak overlap becomes more serious at lower energies (below $\sim 1 \mathrm{keV}$ ), the poor energy resolution of the conventional XEDS detector is one of its essential limitations, especially for light element analysis. Recently, new types of X-ray detectors with much higher energy resolutions have been developed $[1,2]$. However, the conventional XEDS detectors must still be used until the newer detectors are commonly available. In this paper an approach to improve the energy resolution of a conventional XEDS spectrum is presented.

The energy resolution of an XEDS detector is described as a quadrature addition of the intrinsic peak width and the electronic noise during the detection process [3]. The intrinsic peak width is the theoretical resolution limit of detection and is dependent on the incoming X-ray energy $(\sim 110$ $\mathrm{eV}$ at the $\mathrm{MnK} \alpha$ peak for a $\mathrm{Si}(\mathrm{Li})$ detector). The electronic noise is the major source of the difference between the practical and theoretical energy resolutions and the width of the electronic noise depends on the clock time of counting. In some XEDS systems, the electronic noise can be displayed as a strobe peak at the zero energy position [4] (note that the strobe peak may be hidden by the operation software and/or never be seen in many systems). So, the electronic noise (the zero strobe peak) can be regarded as the spread function of the detector and can be deconvoluted from the original XEDS spectrum to improve the energy resolution. The deconvolution is just an inverse operation of the convolution mathematically. However, direct deconvolution is more sensitive to random noise components in the data and the results are practically meaningless. Recently, many deconvolution processes have been developed to improve the resolution of spectra and images [5]. In this study, the Richardson-Lucy algorithm, which is based on an iterative convolution process, was applied to the deconvolution of XEDS spectra, using the zero strobe peak as the spread function. The artifacts induced by the iteration process were minimized by auto- and cross-correlation prefilterings of the spread function [6].

Figure 1 shows a comparison between conventional and deconvoluted spectra taken from a National Institute of Standards and Technology thin-film glass standard reference material (SRM) 2063 [7] in a VG HB 603 analytical electron microscope (AEM). As shown in Fig. 1, all the major peaks become sharper and narrower, including the zero strobe peak. In addition, several minor peaks such as $\mathrm{CK} \alpha$ and FeL $\alpha$ become visible after deconvolution. From the original and deconvoluted spectra, the full width at half maximum (FWHM) energy resolutions of 5 major peaks $(\mathrm{OK} \alpha, \mathrm{MgK} \alpha, \mathrm{SiK} \alpha, \mathrm{CaK} \alpha$ and $\mathrm{FeK} \alpha)$ were determined and plotted as a function of the X-ray energy in Fig. 2. By the zero-peak deconvolution, the energy resolutions of all the peaks are improved. In particular, the improvement in the energy resolution of the OK $\alpha$ peak is $\sim 4$ times (from $\sim 120 \mathrm{eV}$ to $\sim 30 \mathrm{eV}$ ). The solid lines in Fig. 2 indicate the energy resolutions calculated using the width of the zero strobe peak. It can be seen that the zero-peak deconvolution is more effective for peaks in the lower energy region. The calculated energy resolution of the deconvoluted spectrum is $\sim 115 \mathrm{eV}$ at the $\mathrm{MnK} \alpha$ peak, which is very close to the theoretical limit. Figure 3 shows the ratio of the $\mathrm{P} / \mathrm{B}$ ratio of the original spectrum to that of the convoluted spectrum for the major peaks. The $\mathrm{P} / \mathrm{B}$ ratio is more improved at lower energies. So, this method will improve the analytical sensitivity especially for lower energy X-ray lines. The zero-peak deconvolution method was applied to a spectrum from $\mathrm{TiO}_{2}$, in which overlap of 
OK $\alpha$ with TiL $\alpha$ is always unavoidable when using XEDS. The original and deconvoluted spectra of $\mathrm{TiO}_{2}$ are shown in Fig. 4. After deconvolution, the TiL $\alpha$ peak, hidden in the unsymmetrical shoulder of OK $\alpha$ peak, appears. Note that proper background subtraction may be required prior to the deconvolution process in order to minimize artifacts.

In conclusion, zero-peak deconvolution is very useful especially for characteristic X-rays in the lower energy region of the spectrum and this approach is also expandable to spectrum imaging.

\section{References}

[1] D.A. Wollman et al., J. Microscopy, 199 (2000), 37.

[2] M. Terauchi et al., J. Electron Microscopy, 50 (2001), 101.

[3] D.B. Williams and C.B. Carter, Transmission Electron Microscopy, Plenum, New York, 1996.

[4] S.J.B. Reed, Electron Microprobe Analysis, 2nd ed., Cambridge Univ. Press, Cambridge, 1993.

[5] P.A. Jansson ed., Deconvolution of Image and Spectra, Academic Press, San Diego, 1997.

[6] P.B. Crilly, IEEE Trans. Instrum. Meas., 40 (1991), 558.

[7] Certificates of Analysis for SRM 2063. Microanalysis Thin Film Mg-Si-Ca-Fe, Standard Reference Materials Program, NIST, Gaithersburg, MD20899.

[8] This research was supported by Bechtel Bettis Laboratories and NSF DMR 99-72670.
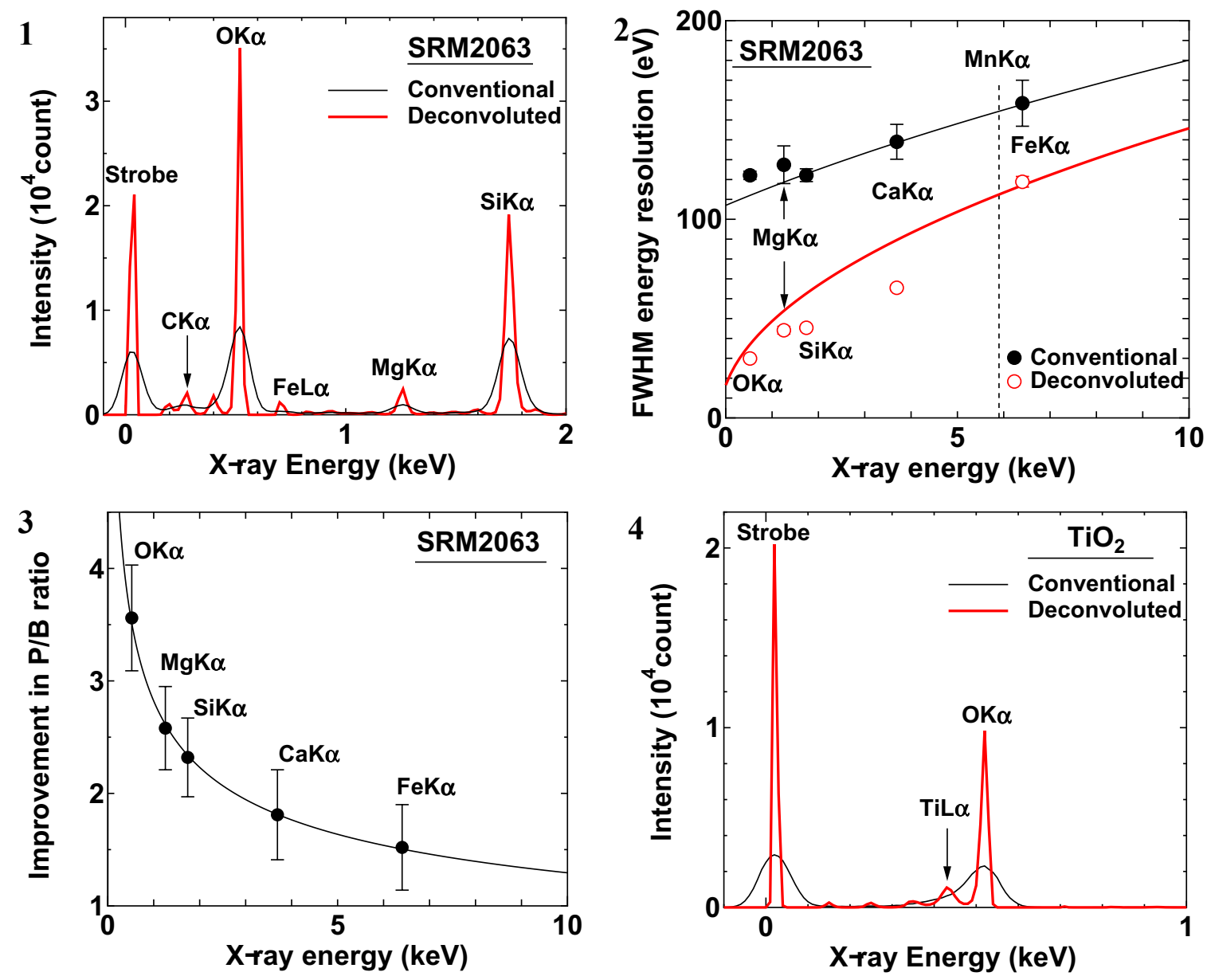

Fig. 1 Comparison of the original and deconvoluted XEDS spectra from a SRM2063 specimen.

Fig. 2 Improvements in the energy resolutions of major peaks in SRM2063 by the deconvolution.

Fig. 3 Improvements in the P/B ratio of the major peaks in SRM2063 by the deconvolution.

Fig. 4 Original and deconvoluted XEDS spectra from $\mathrm{TiO}_{2}$. By deconvoluting the strobe peak, the hidden TiL $\alpha$ peak becomes visible. 